\title{
ASPECTOS ÉTICOS Y LEGALES DE LA INVESTIGACIÓN CIENTÍFICA EN BRASIL
}

\author{
Paulo Roberto da Silva*, Roberto Carlos Barrera García**, Rudney da Silva***
}

\begin{abstract}
Resumen: Este artículo buscó investigar y analizar el proceso ético regulatorio de la investigación científica en Brasil. Fue realizado a través de una revisión sistemática, caracterizada como estudio bibliográfico y documental, siendo los resultados analizados con la técnica de análisis de contenido. El material jurídico levantado trata principalmente de las garantías constitucionales asociadas con la investigación científica, del sistema de regulación ética, de la definición de usuario, de las investigaciones clínicas, de las investigaciones con cooperación extranjera, de las garantías del tratamiento y diagnóstico consolidados, de las investigaciones en reproducción humana, con poblaciones aborígenes, en genética humana, de los proyectos de multicéntricos y de las bases de datos biológicas. Se puede concluir que existen leyes de protección a la investigación científica en el Brasil, estableciendo prioridades nacionales, determinando derechos y deberes de investigadores y de los sujetos de investigación. Existe un sistema nacional de regulación ética establecido por resoluciones del Poder Ejecutivo. Las resoluciones incluyen temáticas asociadas con la investigación científica, manifestándose desde las directrices mundiales acerca de la ética en investigaciones, en este caso la declaración de Helsinki.
\end{abstract}

Palabras clave: ciencia, investigación, bioética, legislación, jurisprudencia

\section{ETHICAL AND LEGAL ASPECTS OF SCIENTIFIC RESEARCH IN BRAZIL}

\begin{abstract}
This article has searched and analyzed the regulatory process of the ethics of scientific research in Brazil. The methodology consisted in a systematic review, characterized by a bibliographical and documental study, results being analyzed by the content analysis technique. Legal documents studied dealt mainly with constitutional guaranties associated with scientific research, system of ethics regulation, definition of user, clinical investigations, investigations with foreign cooperation, treatment and diagnosis safeguards, human reproduction research, research with aboriginal populations, human genetics research, multicenter projects and biological data bases. It can be concluded that there are protection laws for scientific research in Brazil, establishing national priorities, determining researchers and research subjects rights and duties. There is a national system of ethics regulation established by resolutions of legislative power. Resolutions include diverse topics associated with scientific research, in line with world guidelines for research ethics, in this case the Helsinki Declaration.
\end{abstract}

Key words: science, research, bioethics, legislation, jurisprudence

\section{ASPECTOS ÉTICOS E LEGAIS DA PESQUISA CIENTÍFICA NO BRASIL}

Resumo: Este artigo buscou investigar e analisar o processo ético regulatório da pesquisa científica no Brasil. Foi realizado por meio de uma revisão sistemática, caracterizado como estudo bibliográfico e documental, sendo os resultados analisados por meio da técnica de análise de conteúdo. O material jurídico levantado trata principalmente, das garantias constitucionais associadas à pesquisa científica, do sistema de regulação ética, da definição de usuário, das pesquisas clínicas, das pesquisas com cooperação estrangeira, das garantías do tratamento e diagnóstico consolidados, das pesquisas na reprodução humana, das pesquisas com populações indígenas, das pesquisas em genética humana, dos projetos multicêntricos e das bases de dados biológicas. Pode-se concluir que existem leis de amparo à pesquisa científica no Brasil, estabelecendo prioridades nacionais, determinando direitos e deveres dos pesquisadores e dos sujeitos de pesquisa. Existe um sistema nacional de regulação ética; todavía estabelecido por resoluçóes do poder executivo. As resoluçóes incluem diversas temáticas associadas com a investigação científica, incluindo as diretrizes mundiais acerca da ética em pesquisa, considerando até a Declaração de Helsinki.

Palavras-chave: ciencia, investigação, bioética, legislação, jurisprudencia

* $\quad$ Lic. en Direto, Investigador, Universidad do Vale del Itajaí, Brasil

Correspondencia: pauloroberto-adv@hotmail.com

** Lic. en Educación Física, Investigador, Universidad Metropolitana de Ciencias de la Educación, Brasil

Correspondencia: fisioex@gmail.com

*** Lic. en Educación Física, Investigador, Universidad del Estado de Santa Catarina, Brasil

Correspondencia: rudney@udesc.br 


\section{Introducción}

La investigación científica ha crecido exponencialmente en todo el mundo en las últimas décadas. Estudios han demostrado que Estados Unidos de América, la Comunidad Europea y Japón poseen más de dos tercios del total de la producción científica actual. Brasil se ha destacado en el escenario mundial, teniendo el $8 \%$ del total de conocimiento producido, ocupando la $9^{a}$ posición entre los países con mayor dinamismo de crecimiento y la 17 a posición mundial $(1,2)$.

Uno de los factores que legitiman el crecimiento científico es la creación, en las dos últimas décadas, de legislación que fomenta, prioriza y promueve el desarrollo científico y tecnológico, determinando inclusive tratamiento prioritario a la investigación básica en Brasil. No obstante, a pesar de que el aumento de investigaciones científicas en Brasil no se limita a una única ciencia o área de conocimiento humano, se verifica la acentuación en el crecimiento de las investigaciones tecnológicas y relacionadas con las ciencias biológicas y la salud.

Este fenómeno ocurre por factores políticos, sociales y principalmente económicos, pues el modo de producción de conocimientos se relaciona con el de producción de bienes y servicios $(3,4)$, principalmente en las universidades, exigiendo así que se dedique escasa atención a los derechos humanos en el ámbito de esta institución(5).

Entendiéndose que el modo productivo no tiene como prioridad la dignidad de la persona humana, se revela así la necesidad de un sistema ético de control social de la investigación científica, con amplio apoyo legal, que no adopte aptitudes exclusivamente restrictivas, pero que tampoco presente posturas ingenuas o simplistas sobre el proceso de producción del conocimiento humano.

Considerando lo expuesto, este artículo tiene como objetivo investigar y analizar el proceso ético regulatorio de la investigación científica en el Brasil. Expone las leyes fundamentales y complementarias de la legislación brasileña sobre la investigación científica, analiza las resoluciones que establece el sistema brasileño de regulación ética de la investigación y discute las características del desarrollo científico brasileño.

\section{Método}

Este artículo se caracterizó como un estudio bibliográfico y documental, realizado por medio de una revisión sistemática, principalmente en fuentes primarias de datos documentales y bibliográficos $(6,7)$.

La identificación, selección, análisis y discusión de los materiales compilados fue orientada por un cuadro operacional de indicadores construidos para este trabajo(7). Fueron seleccionados y analizados artículos científicos y reglamentaciones disponibles en Internet. Se desarrolló en cuatro etapas: 1) identificación y selección de la base de los datos científicos; 2) selección de artículos sobre los temas seleccionados; 3) identificación y selección de la base de datos jurídicos, y 4) levantamiento y análisis de las legislaciones y reglamentaciones brasileñas.

La primera etapa procuró la selección de los sitios/bases de datos que atendiesen a tres criterios de inclusión: ser caracterizados como una base de datos científicamente confiables; ofrecer gratuita e integralmente materiales electrónicos y ser presentados en lengua portuguesa. De este modo, se seleccionó la base de datos Scientific Electronic Library Online - SciELO, una biblioteca electrónica que envuelve una colección seleccionada de periódicos científicos brasileños, permitiendo así identificar las citaciones en sus artículos.

La segunda buscó artículos que dieran soporte para la discusión teórica al estudio, por medio de los descriptores "ética" y "producción científica”, identificando 53 artículos. A partir de esta selección, se realizó un análisis preliminar de artículos que atendiesen básicamente a dos criterios: fecha de publicación entre enero de 2005 y enero de 2009 y relevancia del artículo a las temáticas investigadas. Este procedimiento permitió identificar seis estudios relevantes a la investigación.

La tercera buscó la selección de bases de datos jurídicos que atendiesen a dos criterios de inclusión: ser caracterizada como una base de datos o sitio electrónico oficial del gobierno brasileño y disponer gratuita e íntegramente normas y leyes en formato electrónico. De esta manera, se seleccionó el sitio electrónico de la Comisión Nacional de Ética en Investigación y la base de la Legislación Federal de Brasil.

La cuarta etapa buscó el levantamiento y análisis de las resoluciones y leyes brasileñas. La selección ocurrió por medio de tres pasos; 1 ) búsqueda simple de reglamentación por medio de los descriptores "ética", "investigación" y "ciencia”, lo que permitió la identificación de 42 documentos; 2) análisis preliminar de las reglamentaciones que atendiesen básicamente a 
dos criterios de análisis: a) relevancia del artículo de acuerdo con los descriptores investigados; b) presencia de términos relacionados con los descriptores investigados, procedimiento que permitió seleccionar 28 documentos; 3) estudio minucioso de la legislación por medio de análisis crítico de los contenidos presentados, procedimiento que permitió identificar 19 documentos, agrupados en tres estratos: a) legislaciones fundamentales ( $\mathrm{n}=01$ casos); b) legislaciones complementarias ( $\mathrm{n}=03$ casos); $\mathrm{c}$ ) resoluciones ( $\mathrm{n}=15$ casos).

Las informaciones fueron analizadas con apoyo del cuadro operacional, utilizando las herramientas de unidades de significancia y reagrupamiento temático de la técnica de análisis de contenido(8).

\section{Resultados}

\section{Ciencia y legislación fundamental}

El levantamiento documental permitió identificar siete Constituciones del Estado brasileño anteriores a la actual, incluyendo sus enmiendas y alteraciones realizadas por asambleas constituyentes o imposiciones de actos institucionales. Así, se constató que la ciencia fue tratada de diferentes maneras a través los tiempos, siendo considerada desde apenas una única garantía de enseñanza en los colegios y en las universidades(9), pasando por todas las restricciones posibles en un gobierno militar(10) hasta ser considerada una prioridad del gobierno brasileño(11).

La Constitución actual de la República Federativa de Brasil(11), promulgada en 1988, después del último gobierno militar, establece en sus principios fundamentales que el país se constituye en un Estado democrático de derecho que tiene como fundamento la dignidad de la persona humana (artículo $1^{\circ}$, inciso III) y que se asegura la libre expresión de la actividades intelectuales y científicas, independientemente de censura o permiso (artículo 5, inciso IX).

En relación con el sistema único de salud, además de otras atribuciones previstas en los términos de la ley magna(11), se constata la obligación de incrementar en su área de actuación el desarrollo científico y tecnológico (artículo 200, inciso V). Respecto de la enseñanza superior, se verifica que las universidades gozan de autonomía didáctico-científica y que deben obedecer al principio de indisociabilidad entre enseñanza, investigación y extensión, cabiéndoles todavía la posibilidad de admisión de científicos extranjeros, bajo la forma de la ley (artículo 207, parágrafo 1º). Para esto, se prevé que la ley establecerá el plan nacional de educación de duración plurianual, procurando la articulación y el desarrollo de la enseñanza en sus diversos niveles y la integración de las acciones del poder público que conducirán a, entre otros, la promoción científica y tecnológica (artículo 214, inciso V), estableciendo que constituyen patrimonio brasileño las creaciones científicas y tecnológicas (artículo 216, inciso III), así como los conjuntos urbanos y sitios de valor científico (artículo 216, inciso V).

La Constitución Federal de 1988 (11) dedica el capítulo $\mathrm{V}$ específicamente a la ciencia y tecnología. Para esto, se constata que el Estado promoverá e incentivará el desarrollo científico, la investigación y la capacitación tecnológica y que la investigación científica básica recibirá tratamiento prioritario del Estado para el progreso de las ciencias (artículo 218, parágrafo $1^{\circ}$ ). De esta forma, determina que la investigación tecnológica deberá dedicarse preponderantemente a la solución de problemas brasileños y al desarrollo del sistema productivo nacional y regional (artículo 218, parágrafo $2^{\circ}$ ).

La formación de recursos humanos en las áreas de ciencias, investigación y tecnología, prevista en la Constitución brasileña(11), será apoyada por el Estado, que concederá medios y condiciones especiales de trabajo a los que de ella se ocupen (artículo 218, parágrafo $3^{\circ}$ ). Serán estimuladas las empresas que inviertan en la investigación y en la creación de tecnología adecuada al país (artículo 218, parágrafo 4ㅇ), así como es facultativo de los Estados y del Distrito Federal destinar parte de sus recursos a las investigaciones científicas y tecnológicas (artículo 218, parágrafo 5). El mercado interno, que integra el patrimonio nacional, será incentivado de modo de viabilizar la autonomía tecnológica del país (artículo 219).

\section{Ciencia y legislaciones complementarias}

El Código Penal brasileño(12), aprobado en 1940 y que entró en vigor a partir de 1942 hasta la actualidad, determina que no constituye injuria o difamación la opinión desfavorable de, entre otras, la crítica científica (artículo 142, inciso II). Acerca de la protección a la vida y a la dignidad, que pueden se afectadas por investigaciones científicas, se constata que la acción de provocar la muerte de alguien es crimen punible con reclusión y que será calificado si es cometido por 
otro motivo "torpe" o "banal" (artículo 121); prevé detención provocar lesión corporal a través de la ofensa a la integridad corporal o a la salud de terceros. La lesión corporal grave, que resulte en incapacidad para ocupaciones habituales, peligro de vida, debilidad permanente de miembros, sentidos o funciones, y si resulta en incapacidad permanente para el trabajo, enfermedades incurables, pérdida o inutilización de miembro, sentido o función, deformidad permanente o aborto, es punible con reclusión (artículo 129, parágrafo $2^{\circ}$, inciso $\left.\mathrm{I}-\mathrm{V}\right)$.

El Código de Propiedad Intelectual de 1996(13) establece que no se considera invención ni modelo de utilidad, entre otros, los descubrimientos de teorías científicas y métodos matemáticos (artículo 10, inciso I), y obras científicas (artículo 10, inciso IV). Con relación a la patente, su titular no tiene derecho de impedir a terceros no autorizados utilizarla con finalidad experimental relacionada con estudios o investigaciones científicas o tecnológicas (artículo 43, inciso II). No son registrables como marcas, entre otros, obras científicas $\mathrm{y}$ títulos protegidos por derechos de autor y que sean susceptibles de causar confusión o asociación, salvo con consentimiento del autor o titular (artículo 124, inciso XII), ni otro termino técnico usado en la ciencia que tenga relación con el producto o servicio a distinguir (artículo 124, inciso XIII). Se constata que el titular de la marca no podrá impedir la citación de la marca en una obra científica o en cualquiera otra publicación si se usa sin connotación comercial y sin daños para su carácter distintivo (artículo 132, inciso IV).

El Código Civil de 2002(14) determina que es válida, con objetivos científicos o altruistas, la disposición gratuita del propio cuerpo, en todo o en parte, para después de la muerte (artículo 14). El código no considera empresario a quien ejerce profesión intelectual de naturaleza científica, así como con el concurso de auxiliares o colaboradores, salvo si el ejercicio de la profesión constituye elemento de empresa en términos de las leyes (artículo 966, parágrafo único).

\section{Ciencia, bioética y normas reglamentarias}

\section{Sistema ético-regulatorio}

La Resolución no 001/1988/CNS(15) regulaba la acreditación de centros de investigación en Brasil y recomendaba la creación de comités de ética en investigación en cada localidad del país. Esta resolución trataba de investigación científica, de ética y sus implicaciones, de las competencias de los órganos estatales, de las empresas privadas y de los investigadores, de las exigencias institucionales, físicas y humanas para la realización de la investigación científica, de la creación de la figura del comité de ética, de los derechos y deberes del investigador y del investigado -principalmente menores de edad, mujeres, individuos con capacidad reducida de autonomía (estudiantes, militares, entre otros), incluyendo parte o sus componentes orgánicos-, así como también contemplaba las investigaciones que involucraban procedimientos radioactivos, entre otros aspectos.

La Resolución no 170/1995/CNS(16) propone la creación de un grupo ejecutivo de trabajo del Ministerio Brasileńo de Salud, conectado con el Consejo Nacional de Salud, para revisar y actualizar la Resolución no 001/1988/CNS(15), además de designar renombrados miembros de las áreas relacionadas con la investigación científica en Brasil, incluyendo representantes de la Comisión Intersectorial de Ciencia y Tecnología del Consejo Nacional de Salud y de la Confederación Nacional de la Industria.

La Resolución no 173/1995/CNS(17) buscaba determinar que el proceso de revisión y actualización de la Resolución de 1988 fuera desarrollado en observancia del plan de trabajo aprobado por el Plenario del Consejo Nacional de Salud, que tenía por objeto: la revisión de los aspectos éticos de la investigación en salud con seres humanos, objetivando la actualización y buscando resolver las lagunas existentes en dicha resolución; la normalización separada de algunos campos temáticos de alta relevancia y que no estaban siendo contemplados o se encontraban mezclados en la resolución; definiciones sistemáticas de su operacionalización, asegurando los mecanismos de implantación, implementación y acompañamiento de las normas de investigación en salud que incluían seres humanos. El plan preveía también los procedimientos a seguir.

La Resolución no 196/1996/CNS(18) aprueba las directrices y normas reglamentarias de las investigaciones que involucran seres humanos en Brasil, creadas por el grupo de trabajo instituido por las resoluciones $\mathrm{n}^{\text {os }} 170 / 1995 / \mathrm{CNS}(16)$ y $173 / 1995 / \mathrm{CNS}(17)$. Para esto, presenta términos y definiciones necesarias para la normalización de la nomenclatura; aborda los aspectos éticos de la investigación que incluye seres humanos, así como también da garantías fundamentales al sujeto de 
investigación; ofrece informaciones detalladas sobre la elaboración y obtención de consentimientos informados, inclusive con una relación de los ítems básicos que deben componer este instrumento; detalla los riesgos y formas de reducirlos, además de los aspectos relacionados con los beneficios de la investigación científica con seres humanos; define los criterios necesarios para la presentación, apreciación, análisis y aprobación de los protocolos de investigación, e informa la necesidad de someter las investigaciones con seres humanos a un comité de ética de la investigación acreditado por la Comisión Nacional de Ética en Investigación, estableciendo su organización, composición y funcionamiento, incluyendo sus atribuciones y actuación. Crea también la Comisión Nacional de Ética en Investigación y define su composición y atribuciones. Por último, presenta la operacionalización inherente a los investigadores, comités de ética y Comisión Nacional de Ética en Investigación.

La Resolución no 370/2007/CNS(19), complementando la no 196/1996/CNS(18), trata del registro, acreditación y renovación de los comités de éticas brasileños. Establece los procedimientos y condiciones mínimas para este fin, principalmente en lo que se refiere a las reglas operacionales de protocolización, recibimiento, manipulación, análisis, parecer y almacenamiento de los protocolos sometidos al Comité de Ética en Investigación, además de las condiciones humanas, físicas, organizacionales, materiales y de información. Determina plazos de regularización, composición, vinculación administrativa y garantía de ausencia de conflictos de interés de cualquier tipo y de actuación independiente y autónoma de los miembros de los comités de ética vinculados al sistema.

\section{Definición de usuario}

La Resolución no 240/1997/CNS(20), complementando la no 196/1996/CNS(18), resuelve que el término "usuario" se aplica a una interpretación amplia, contemplando colectividades múltiples que se benefician con el trabajo desarrollado por la institución, y que los representantes de usuarios son personas capaces de expresar puntos de vistas e intereses de individuos y/o grupos sujetos de investigación de determinada institución. Esta resolución establece también que los representantes de usuarios piensen en los intereses colectivos y públicos diversos, en instituciones de referencia para públicos o patologías específicos, y que deben necesariamente pertenecer a la población-objetivo de la unidad o al grupo organizado que defienda sus derechos. En los locales donde existan foros o consejos de entidades representativos de usuarios portadores de patologías y deficiencias, cabe a estas instancias indicar los nombres de los representantes de usuarios para los comités de ética en investigación, los cuales deben ser informados al Consejo Municipal correspondiente.

\section{Investigación clínica}

La resolución no 251/1997/CNS(21), complementando la no 196/1996/CNS(18), aprueba normas de investigación con seres humanos para el área temática de investigación con nuevos fármacos, medicamentos, vacunas y pruebas diagnósticas. Para esto, establece que deben ser respetadas las normas, resoluciones y leyes pertinentes del poder público. Determina también los cuidados necesarios para la preservación de la dignidad y el bienestar de los sujetos de investigación, y las bases científicas necesarias para el desarrollo de este tipo de investigación. Dedica atención a las exigencias necesarias para que nuevos productos constituyan avance significativo respecto de los ya existentes. Presenta términos y definiciones necesarias a la normalización científica, a las responsabilidades del investigador y a las exigencias de los protocolos de investigación sobre nuevos fármacos, medicamentos, vacunas y pruebas diagnósticas. Define también las atribuciones del Comité de Ética en Investigación y la forma de operacionalización del Comité, inherente a la Comisión Nacional.

\section{Cooperación extranjera}

La Resolución no 292/1999/CNS(22) complementaba la no 196/1996/CNS(18) y está actualmente anulada. Esta resolución definía cómo entender la cooperación extranjera y excluía ciertas investigaciones que no entraban en esta área temática especial. Determinaba también que los gastos y beneficios inherentes a la investigación debían ser distribuidos equitativamente entre los involucrados, así como la necesidad de atención a las normas y disposiciones legales sobre envío de materiales al exterior y a las que protegen la propiedad industrial y/o transferencia tecnológica, además de todas las implicaciones de la investigación científica de esta área temática especial. 


\section{Garantías de tratamiento y diagnóstico consolidados}

La Resolución no 301/2000/CNS(23) establece la mantención integral de la garantía del mejor tratamiento diagnóstico o terapéutico comprobado en cualquier estudio médico y a todos los pacientes, incluyendo aquellos del grupo control. Se manifiesta contraria a las alteraciones, sobre todo a aquellas referentes al uso de placebo, frente a la existencia de métodos diagnósticos y terapéuticos comprobados. Solicita también a la Sociedad Médica Brasileña que la posición expuesta sea remitida a los organizadores de la Asamblea General de la Asociación Médica Mundial.

La Resolución no 404/2008/CNS(24) determina que al final del estudio todos los pacientes participantes deben tener asegurado el acceso a los mejores métodos comprobados, profilácticos, diagnósticos y terapéuticos, identificados por el estudio. Sostiene también que beneficios, riegos, dificultades y efectividad de un nuevo método deben ser probados comparándolos con los mejores métodos profilácticos, diagnósticos y terapéuticos actuales, sin que se excluya el uso de placebo o tratamiento en estudios donde no existan métodos probados de prevención, diagnósticos o tratamiento. La resolución finaliza con la propuesta de que se expanda el acceso a cuidados de salud y a los productos que se presenten eficaces para todos los que lo necesiten.

\section{Reproducción humana}

La Resolución no 303/2002/CNS(25), complementando la no 196/1996/CNS(18), aprueba normas sobre investigación en reproducción humana, presentando las atribuciones del Comité de Ética en Investigación sobre reproducción humana asistida, anticoncepción, manipulación de gametos, preembriones, embriones y fetos, además de medicina fetal, así como las implicaciones relacionadas con este tipo de investigación. Determina también que el Comité de Ética debe examinar el protocolo, elaborar el parecer y enviar ambos a la Comisión Nacional de Ética en Investigación con la documentación completa, conforme se establece en la Resolución no 196/96/CNS(18), correspondiendo a esta instancia federal la aprobación final del protocolo. Queda delegada al Comité de Ética la aprobación de las investigaciones en otras áreas de reproducción humana. Establece que en estas investigaciones serán considerados "sujetos de investigación" todos los afectados por los procedimientos de la misma.

\section{Poblaciones indígenas}

La Resolución no 304/2002/CNS(26), complementando la no 196/1996/CNS(18), aprueba normas sobre investigación en pueblos indígenas. Para esto presenta términos y definiciones necesarias, destacando diferentes aspectos éticos de esta clase de investigación y sus implicaciones físicas, psíquicas y sociales. Define las exigencias necesarias de estos protocolos, además de enfatizar en la protección de los implicados. Presenta también las atribuciones de la Comisión Nacional de Ética en Investigación y de los comités de ética catastrados, además de la Comisión Intersectorial de Salud del Indio.

\section{Genética humana}

La Resolución no 340/2004/CNS (27), complementando la $\mathrm{n}^{\circ} 196 / 1996 / \mathrm{CNS}(18)$, presenta términos y definiciones de la genética humana, destacando diferentes aspectos éticos de esta clase de investigación, las exigencias de los protocolos de investigación en esta área temática especial, la elaboración y obtención del consentimiento informado, además de las atribuciones y funcionamiento de los comités de ética y de la Comisión Nacional de Ética en Investigación. Esta resolución contempla también diversos aspectos polémicos sobre genética humana y que son constantemente asociados con la discusión ética.

\section{Proyectos multicéntricos}

La Resolución no 346/2005/CNS (28), complementando la no 196/1996/CNS(18), anula la reglamentación de la resolución 292/1999/CNS(22). Para esto presenta una definición de lo que la Comisión Nacional de Ética en Investigación entiende como proyectos multicéntricos, informando sobre la tramitación de protocolos en esta área y presentando procedimientos de envío, análisis y aprobación de los protocolos en los comités de ética en investigación y en la Comisión Nacional. Además, la relación y atribuciones de los centros participantes, el envío del parecer final y las implicaciones de las ocurrencias de evento adverso.

\section{Banco de datos biológicos}

La Resolución no 347/2005/CNS (29), complementando la no 196/1996/CNS, establece que para proyectos de investigación que busquen el almacenamiento de materiales biológicos humanos para investigaciones 
futuras, además de los puntos previstos en la Resolución no 196/1996/CNS(18), deben ser atendidas las directrices específicas para análisis éticos de proyectos de investigación de esta área, presentar la norma o el reglamento de la institución depositaria para el almacenamiento de materiales biológicos humanos, basados en los ítems identificados en la resolución, el período de almacenamiento, el papel de las instituciones implicadas y, en caso de almacenamiento o formación del banco de material biológico en el exterior, la obediencia a la legislación vigente, además del número de cotas del investigador brasileño y los procedimientos para el uso futuro de las muestras almacenadas.

\section{Discusión}

En Brasil existe una pirámide jurídica legal que define la jerarquía de las normas, siendo la Constitución Federal la considerada la Ley Máxima y las demás normas como infrainstitucionales(30). Considerando que la Constitución de un país es su ley máxima(31), se puede constatar que la ciencia es una actividad legalmente garantizada en Brasil, posibilitando investigaciones que pueden variar entre diversas temáticas. Entretanto, el ejercicio de la investigación no exime al científico de las responsabilidades inherentes a la protección de la dignidad de la vida humana, responsabilizando así a todos los que abusaren de sujetos humanos. Se constata también que la ciencia básica y la investigación tecnológica son prioridades gubernamentales del Estado brasileño.

Considerando las garantías fundamentales previstas en las leyes, no se pueden ignorar los antagonismos entre un modo de producción de bienes y servicios y el modo de producción de conocimientos, que en el Brasil sucede casi exclusivamente en las universidades(32:8-14). Asimismo, deben tenerse en cuenta las investigaciones de organismos privados, por ejemplo aquellos vinculados a las investigaciones clínicas de nuevos fármacos. Se puede afirmar que la universidad se viene convirtiendo en rehén de las directrices impuestas por el mercado, ya que se sustenta económicamente en el modelo actual que exige soluciones prácticas(32:6).

Ya en la década de los 80, este fenómeno colocaba a la universidad en una posición bastante incómoda porque, considerando su función técnica y crítica frente a la producción de conocimientos, comprometía su propia existencia independiente. Se destacan en este fenómeno el modelo económico vigente, las exigencias del mercado, la desvalorización de los conocimientos básicos, la sobrevaloración de los conocimientos aplicados y la exacerbada consideración del conocimiento tecnológico, entre otros(3).

Al llevar a la universidad a producir con mayor énfasis soluciones prácticas -al modo de producción de bienes y servicios-, el poder económico obliga a la universidad a dejar de fomentar y promover la producción de conocimientos científicos básicos, principalmente aquellos que supuestamente no interesan al modo de producción.

Esta discordancia entre las exigencias del mercado y las de la universidad restringe el acceso al conocimiento científico y tecnológico a una parte estrecha de la población, pudiendo inclusive llevar a la futura desestructuración de las instituciones sociales y a la disminución del estatus de dignidad de la vida humana.

Es necesario que exista un sistema ético regulatorio que otorgue garantías de respeto y de protección del sujeto humano de investigación a partir de organismos de control social. Este papel intransferible del Estado brasileño debe ser ejercido por todos los implicados en el proceso de producción científica, pues no se puede pretender que organismos privados -cuyo objetivo es la generación de lucro y acumulación de capital- lo prioricen, dado que su meta principal no se encuentra en el beneficio humano.

Considerando las resoluciones investigadas a través de la revisión sistemática de la literatura jurídica, se puede inferir que en Brasil existe un sistema formal de regulación ética de investigación científica con seres humanos que incluye diferentes temáticas, abordando desde la definición de usuario y sus implicaciones hasta las directrices para el desarrollo de las investigaciones clínicas, que en todas las partes del mundo sufren severas regulaciones. Se abordan también temáticas de interés de soberanía nacional, como la cooperación extranjera en estudios desarrollados en el país, garantías de tratamiento y diagnóstico consolidados, reproducción humana, poblaciones indígenas, proyectos multicéntricos, bancos de datos biológicos y genética humana, los cuales, con los avances biotecnológicos actuales, vienen configurándose como uno de los eventos de acentuada relevancia ético-regulatoria(33). 


\section{Agradecimientos}

Este artículo fue realizado con el apoyo institucional del Comité de Ética en Investigaciones con Seres Humanos vinculado al Gabinete del Rector de la Universidad del Estado de Santa Catarina.
El estudio obtuvo una beca integral de estudios de la Organización de las Naciones Unidas para la Educación, la Ciencia y la Cultura, Unesco, en el Curso de Introducción a la Ética de Investigación en Seres Humanos, del Programa de Educación Permanente en Bioética de la Red Latinoamericana de Bioética, 2007-2008.

\section{Referencias}

1. Castiel LD, Sanz-Valero J. Entre fetichismo e sobrevivência: O artigo científico é uma mercadoria acadêmica? Cadernos de Saúde Pública 2007; 23(12): 3041-3050.

2. Glänzel W, Leta J, Bart T. Science in Brazil. Part 1: A macro-level comparative study. Scientometrics 2006; 67(1): 6786.

3. Bunge M. Ciência e desenvolvimento. São Paulo: EDUSP; 1980.

4. De Tomasi L, Warde MJ, Haddad S. O banco mundial e as politicas educacionais. São Paulo: Cortez; 1988.

5. Amador FS. Ética e direitos humanos na e pela pesquisa e extensão universitárias. Revista do Departamento de Psicologia 2005; 17(2): 109-117.

6. Richardson RJ, Peres JAS. Pesquisa Social: Métodos e técnicas. São Paulo: Atlas; 1999.

7. Laville C, Dione J. A construção do saber: Manual de metodologia da pesquisa em ciências humanas. Porto Alegre: Artes Médicas; 1999.

8. Bardin L. Análise de conteúdo. Lisboa: Ediçôes 70; 2000.

9. Brazil. Constitution Politic of Empire of 1824. Pub. L. $4^{\circ}$ of Laws, License and Imperials Letters, 17 (April 22, 1824) (updated 2009 Mar 11; cited 2009 Feb 1). Available from: http://legislacao.planalto.gov.br/.

10. Brazil. Constitution of Federative Republic of 1969. Pub. L. Official Diary, (October 20, 1969) (updated 2009 Mar 11; cited 2009 Feb 1). Available from: http://legislacao.planalto.gov.br/.

11. Brazil. Constitution of Federative Republic of 1988. Pub. L. Union Official Diary, (October 5, 1988) (updated 2009 Mar 11; cited 2009 Feb 1). Available from: http://legislacao.planalto.gov.br/.

12. Law-Decree 2.848, Penal Code. Pub. L. Union Official Diary, (December 31, 1940).

13. Law 9.279, Intellectual Property Code. Pub. L. Union Official Diary, (May 15, 1996).

14. Law 10.406, Civil Code, Pub. L. Union Official Diary, (January 11, 2002).

15. Resolution 01. Council National Health. (June 13, 1988). (updated 2009 Mar 11; cited 2009 Feb 1). Available from: http://conselho.saude.gov.br/.

16. Resolution 170. Council National Health. (November 9, 1995). (updated 2009 Mar 11; cited 2009 Feb 1). Available from: http://conselho.saude.gov.br/.

17. Resolution 173. Council National Health. (November 9, 1995). (updated 2009 Mar 11; cited 2009 Feb 1). Available from: http://conselho.saude.gov.br/.

18. Resolution 196. Council National Health. (October 10, 1996). (updated 2009 Mar 11; cited 2009 Feb 1). Available from: http://conselho.saude.gov.br/.

19. Resolution 370. Council National Health. (March 10, 2007). (updated 2009 Mar 11; cited 2009 Feb 1). Available from: http://conselho.saude.gov.br/.

20. Resolution 240. Council National Health. (June 5, 1997). (updated 2009 Mar 11; cited 2009 Feb 1). Available from: http://conselho.saude.gov.br/.

21. Resolution 251. Council National Health. (August 7, 1997). (updated 2009 Mar 11; cited 2009 Feb 1). Available from: http://conselho.saude.gov.br/.

22. Resolution 292. Council National Health. (July 8, 1999). (updated 2009 Mar 11; cited 2009 Feb 1). Available from: http://conselho.saude.gov.br/.

23. Resolution 301. Council National Health. (March 16, 2000). (updated 2009 Mar 11; cited 2009 Feb 1). Available from: http://conselho.saude.gov.br/.

24. Resolution 404. Council National Health. (March 8, 2008). (updated 2009 Mar 11; cited 2009 Feb 1). Available from: http://conselho.saude.gov.br/. 
25. Resolution 303. Council National Health. (July 6, 2000). (updated 2009 Mar 11; cited 2009 Feb 1). Available from: http://conselho.saude.gov.br/.

26. Resolution 304. Council National Health. (August 10, 2000). (updated 2009 Mar 11; cited 2009 Feb 1). Available from: http://conselho.saude.gov.br/.

27. Resolution 340. Council National Health. (July 8, 2004). (updated 2009 Mar 11; cited 2009 Feb 1). Available from: http://conselho.saude.gov.br/.

28. Resolution 346. Council National Health. (January 13, 2005). (updated 2009 Mar 11; cited 2009 Feb 1). Available from: http://conselho.saude.gov.br/.

29. Resolution 347. Council National Health. (January 19, 2005). (updated 2009 Mar 11; cited 2009 Feb 1). Available from: http://conselho.saude.gov.br/.

30. Silva FV. A lei processual. Buscalegis (serial online) 2008 (cited 2009 Feb 1);0 (0): (17 screens). Available from: URL: http://www.buscalegis.ufsc.br/revistas

31. Pedra AS. Interpretação e aplicabilidade da Constituição: em busca de um Direito Civil Constitucional. Buscalegis (serial online) 2008 (cited 2009 Feb 1);0 (0): (22 screens). Available from: http://www.buscalegis.ufsc.br/revistas.

32. Singer P. Poder, política e educação. Revista Brasileira de Educação 1994; 1(1): 5-15.

33. Clotet J. Bioética como Ética Aplicada e Genética, Bioetica (serial online) 1997 (cited 2009 Feb 1);5 (2): (12 screens). Available from: http://www.portalmedico.org.br/revista/ind2v5.htm. 